OPEN ACCESS

Edited by:

Cheng Zhong,

Tianjin University, China

Reviewed by:

Yanguang $\mathrm{Li}$,

Soochow University, China

Zhengyu Bai,

Henan Normal University, China

*Correspondence:

Huile Jin

huilejin@wzu.edu.cn

Shun Wang

shunwang@wzu.edu.cn

Specialty section:

This article was submitted to Energy Materials,

a section of the journal

Frontiers in Materials

Received: 25 February 2020 Accepted: 09 April 2020 Published: 16 July 2020

Citation:

Wang L, Li J, LU G, Li W, Tao Q,

Shi $C$, Jin $H$, Chen $G$ and Wang $S$ (2020) Fundamentals of Electrolytes

for Solid-State Batteries: Challenges and Perspectives. Front. Mater. 7:111.

doi: 10.3389/fmats.2020.00111

\section{Fundamentals of Electrolytes for Solid-State Batteries: Challenges and Perspectives}

\author{
Liguang Wang ${ }^{1,2}$, Jun $\mathrm{Li}^{1,2}$, Guolong $\mathrm{Lu}^{1}$, Wenyan $\mathrm{Li}^{1}$, Qiqi Tao ${ }^{1}$, Caihong Shi ${ }^{1}$, Huile Jin ${ }^{1 *}$, \\ Guang Chen ${ }^{1}$ and Shun Wang ${ }^{1 *}$ \\ ${ }^{1}$ Key Laboratory of Carbon Materials of Zhejiang Province, Institute of New Materials and Industrial Technologies, Wenzhou \\ University, Wenzhou, China, ${ }^{2}$ Department of Chemistry and Biochemistry, University of Windsor, Windsor, ON, Canada
}

Compared with traditional lithium-ion systems, solid-state batteries could achieve high safety and energy density. Although great improvements have been made, especially in solid-state electrolytes, fundamental challenges still remain for the solid-state systems in terms of chemistry and mechanics. This review summarizes the fundamental issues in solid-state batteries with a focus on three critical phenomena: (i) the principles of developing high ionic conductors, (ii) structural evolution at chemically unstable electrolyte-electrode interfaces, and (iii) the effects of manufacturing solid-state batteries, including electrode, and electrolyte design. The future perspectives are also outlined to guide the development of solid-state batteries.

Keywords: electrolytes, solid-state batteries (SSBs), challenges, perspectives, fundamental understanding

\section{INTRODUCTION}

Research on safety and high-energy conversion and storage systems has taken center stage in the past decade and will be continuing in the near future owing to the global decarbonization trend (Choi et al., 2019; Yuan and Lu, 2019). In parallel, lithium-ion batteries (LIBs) have been used to power portable electrical devices for 30 years ( $\mathrm{Li}$ et al., 2018). However, the contemporary state-of-the-art commercial LIBs with flammable liquid organic electrolytes cannot satisfy the requirements, especially regarding safety, and power density, of the ever-increasing scale battery applications (Nanda et al., 2018).

Employing solid-state electrolytes (SSEs) can not only address the safety concerns but also enable the application of a metal anode and operation at high voltage. Given the nature of flammable organic electrolytes, a battery can be a fire hazard in case of over-charging or short-circuiting (Zhang H. et al., 2017). Solid electrolytes possess a much higher thermal stability, and this makes the solid-state battery (SSB) one of the best choices for the next generation of batteries. Moreover, inorganic solid electrolytes can work in hostile environments, such as in the temperature range from -50 to $200^{\circ} \mathrm{C}$ or even higher, in which organic electrolytes fail due to freezing, boiling, or decomposition (Zhao et al., 2018). The SSBs based on SSEs could also significantly increase the energy density and the power density, which are the critical features for large energy storage systems. Various SSEs with high lithium-ion conductivity and chemo-mechanical stability were developed in the past decades (Che et al., 2017; Fan et al., 2018). Based on their components, the reported SSEs can be separated into three main categories: inorganic electrolytes, organic electrolytes, and composite electrolytes. Inorganic electrolytes usually exhibit high ion conductivity of over $10^{-4} \mathrm{~S} / \mathrm{cm}$ with high thermal stability owing to their inflexible crystal structures (Wang S. et al., 2018). Some of the reported inorganic electrolytes 
show ionic conductivity that is competitive with or even higher than commercial liquid electrolytes (Miura et al., 2019). However, SSBs with inorganic electrolytes often deliver lower-thanexpected performance caused by the poor interfacial contact between electrolyte and electrode (Han et al., 2017; Famprikis et al., 2019). Organic polymers differ greatly to inorganic ceramics in their chemical and mechanical properties, leading to different battery designs. Organic electrolytes are feasible for flexible battery designs owing to their soft intrinsic features. However, these materials face other issues, such as low $\mathrm{Li}^{+}$ conductivity $\left(<10^{-5} \mathrm{~S} / \mathrm{cm}\right.$ at room temperature), large ionic transfer resistance, and oxidation at high voltages (Kerman et al., 2017; Strauss et al., 2018). Thus, some researchers believe that ceramic/polymer composite electrolytes may solve the ionic conductivity and interfacial contact issues consistently by combining the advantages of two different types of electrolytes.

Nevertheless, there remain a few urgent problems that prevent large-scale applications of SSBs. For instance, there is serious capacity degradation in high-energy-density SSB systems for multiple electrochemical and mechanical reasons (Zheng et al., 2018). Also, the power density related to their high-rate property-fast charge-discharge performanceremains at too low a level to satisfy commercial applications. In addition, the critical phenomenon of the formation of lithium dendrites over long-term cycling induces short-circuit and thermal runaway, introducing serious safety problems. Most of these problems correlate to the lithium-ion diffusion kinetics, electrode-electrolyte interface, and chemo-mechanical contact between various components (see Figure 1; Zhang et al., 2018). Therefore, in this review, we summarize the challenges and their solutions for current SSEs in terms of the three main aspects of electrolyte selection, electrode-electrolyte interface, and the fabrication of solid-state batteries.

\section{CHALLENGES AND SOLUTIONS FOR CURRENT SOLID-STATE ELECTROLYTES}

When applying all-solid-state electrolytes, four specific features are required to achieve the expected electrochemical performance. These features are (i) high ionic conductivity $\left(\sigma_{\mathrm{Li}}^{+}\right.$ $>10^{-4} \mathrm{~S} / \mathrm{cm}$ ); (ii) sufficient mechanical strength and few enough structural defects to prevent lithium dendrite penetration; (iii) low-cost raw resources and facile preparation processes; (iv) low activation energy for lithium-ion diffusion. To address all of these specific requirements of SSEs, researchers are focusing on the three main challenges of searching for high ionic conductivity electrolytes, manufacturing effective electrodes, and optimizing the electrode-electrolyte interface.

\section{Searching for the High lonic Conductivity Electrolytes}

The consensus has been reached at that ceramic materials can exhibit higher ionic conductivity than polymers. Besides, the electrochemical stability window of polymeric electrolytes is relatively low compared to that of inorganic electrolytes. Nevertheless, to achieve the final goal, researchers are paying much attention to inorganic electrolytes for application in allsolid-state batteries (Pfenninger et al., 2019). In a crystalline solid electrolyte, thermal energy drives the migration of lithium ions
(1) Metallic anodes

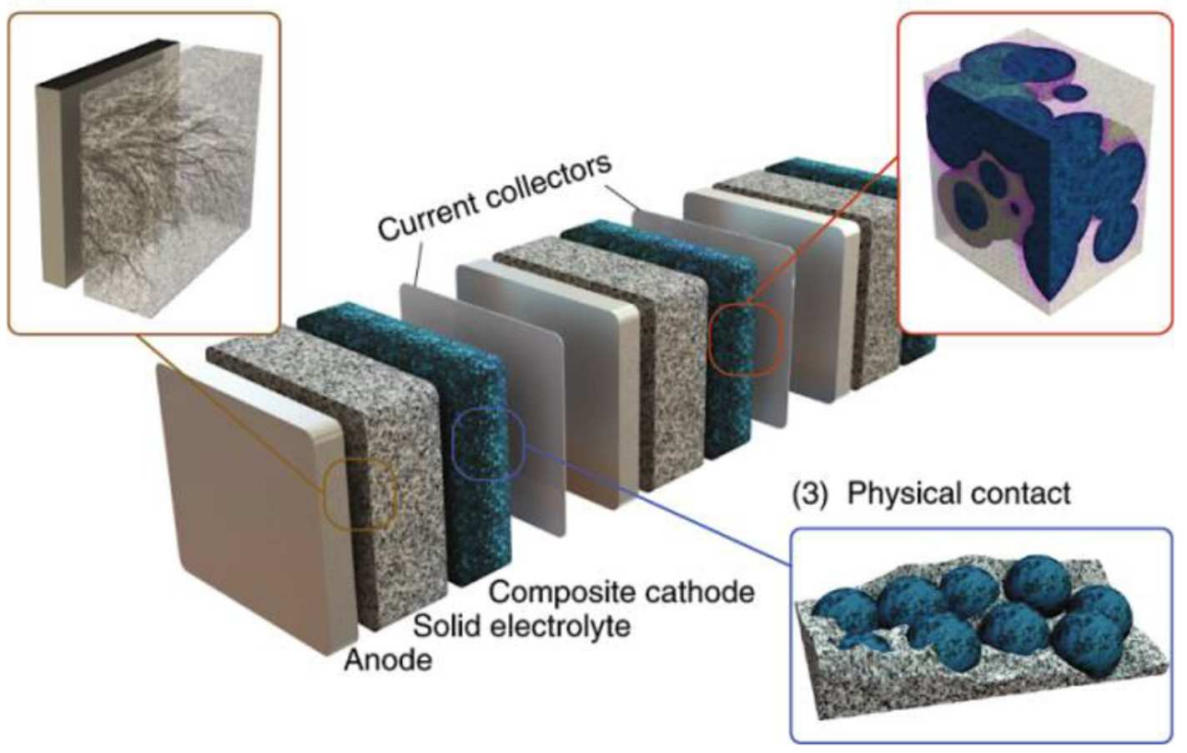

FIGURE 1 | Schematic representation of solid-state batteries. The three main challenges facing SSEs are magnified and highlighted in the three insets: (1) lithium dendrites form when lithium metal is employed as the anode; (2) there is sluggish interface formation, and (3) contact between particles is lost over electrochemical cycling. Reproduced from Famprikis et al. (2019) with permission from Nature Publishing Group. 
through cationic vacancies or interstitials. As we all know, the charge of transfer ions (q), concentration $(n)$, and mobility of charge carriers $(u)$ in the crystal define the ionic conductivity $(\sigma)$, which then can be described by a modified Arrhenius function:

$$
\sigma=q \cdot u \cdot n=\sigma_{0} T^{m} e^{-E_{a} / k_{B} T}
$$

where $\sigma_{0}$ is the pre-factor, $T$ is the temperature, $m$ typically equals $-1, E_{a}$ is a factor of activation energy, including the formation of mobile defects $\left(E_{f}\right)$ and the migration barrier $\left(E_{m}\right)$, and $k_{\mathrm{B}}$ is the Boltzmann constant. According to the basic definition of ionic conductivity (equation 1), in the intrinsic point of view, increasing the concentration of migrating charged species $(n)$ can enhance the ionic conductivity in the solid. Therefore, at a specific battery operating temperature, such as at room temperature, the $n$ value is highly related to the mobile defect formation energy $\left(E_{f}\right)$. Elemental substitution has proved to be one of the best strategies for increasing the charge-compensating vacancies and interstitials. For instance, molecular dynamics (MD) simulations showed that the vacancy concentration can be controlled by changing the quantity of Br-doping in cubic $\mathrm{Na}_{3} \mathrm{PS}_{4}$ (de Klerk and Wagemaker, 2016). Compared to a stoichiometric compound, conductivity an order larger was achieved, with the activation energy reduced from 0.28 to $0.16 \mathrm{eV}$, by only $2 \%$ vacancies. Apart from creating vacancies, ion doping has surprisingly been proved to promote a change in the ionic conductive mechanism. For example, the migration energy of a fast lithium conductor (LISICON-type $\mathrm{Li}_{4 \pm \mathrm{x}} \mathrm{Si}_{1-\mathrm{x}} \mathrm{X}_{\mathrm{X}} \mathrm{O}_{4}, \mathrm{X}=\mathrm{P}, \mathrm{Al}$, or $\mathrm{Ge}$ ) was significantly reduced by extensive ion doping, which then changed the $\mathrm{Li}^{+}$diffusion mechanism from local oscillation to superionic flow, resulting in a "superionic" conductor (Deng et al., 2015, 2017).

\section{Negating Electrode-Electrolyte Interfacial Impedance}

Ceramic electrolytes with a crystalline structure demonstrate better thermal stability and higher ionic conductivity than polymeric materials. Unfortunately, although they have a high bulk conductivity, the formation of grain boundaries in these polycrystalline conductors leads to a large ion migration barrier across the interface. This sluggish ion transfer kinetics makes the diffusion of mobile ions in the electrolyte the rate-determining step, which undermines the advantage of high-power density in SSBs. Therefore, building a good interface between electrode and electrolyte becomes the core task for polycrystalline inorganic electrolytes. For instance, by employing an $\mathrm{ALD}-\mathrm{Al}_{2} \mathrm{O}_{3}$ interlayer on the surface of SSE film, a good interface contact between lithium metal anode and electrolyte was built (Figures 2A,B; Han et al., 2017). Such a well-contacting interface effectively reduced the interfacial area specific resistance from 1,710 to $1 \Omega \cdot \mathrm{cm}^{2}$ (Wang L. et al., 2018b). The challenge of interface impedance is not limited to pristine battery preparation. Loss of contact during cycling owing to inconsistent volume changes between electrolyte and cathode and anode materials is another issue (Figures 2C,D; Tippens et al., 2019). Therefore, electrodeelectrolyte engineering then becomes another key point for SSB fabrication (discussed in the next part) (Wang A. et al., 2018).
Compared to crystalline ceramic materials, polymeric materials and glasses are intrinsically soft and isotropically conductive, resulting in a lack of grain boundaries. From the manufacturing point of view, polymer and glass electrolytes could be easily made into thin films. These thin films can be in good mechanical contact with electrodes, which could dramatically reduce the lithium diffusion resistance in the batteries.

\section{Manufacturing Solid-State Batteries}

Void space in electrode, electrolyte, and electrode-electrolyte interface can promote lithium filament growth, resulting in short-circuit and mechanical degradation. Although many efforts have been made to overcome the chemo-mechanical challenges, the issues caused by the SSE matrix complex and electrode manufacture remain uncovered, which inhibits the large-scale application of SSBs (Kato et al., 2018). Apart from the critical electrode-electrolyte interface, the inevitable voids in the SSE matrix not only reduce the lithium diffusion paths in the SSE but also provide space for lithium dendrite integration (Froboese et al., 2019). Therefore, developing dense electrolyte and electrode with fewer spaces has become another critical approach. Generally, crystalline particles are hard to make into a dense film even under high pressure (Han et al., 2018). To eliminate the void space, many researchers have developed various strategies, such as employing filling materials (mainly small size or soft lithium-ion conductors) into the spaces. On the other hand, quantifying the porosity of the solid-state electrolyte and electrode by using advanced in-situ techniques can also help in the manufacture of the chemo-mechanical structure. X-ray diffraction, especially surface X-ray diffraction techniques, can be applied to analyze the evolution of the crystal structure of battery materials and interfaces. Synchrotron $\mathrm{X}$-ray spectroscopy techniques can provide information on the electronic and local structure of the elements of interest. Further combining spatially resolved imaging techniques with the abovementioned spectroscopic measurements can enable the chemo-mechanical changes in different battery components to be directly observed (Wang J. et al., 2017). For instance, by combining in-situ synchrotron-based X-ray tomography with electrochemical measurements (Wang L. et al., 2017, 2018b), it was possible to identify the degradation mechanisms triggered by the bending and cracking generated in both electrodes and electrolyte over cycling (Zhang W. et al., 2017). High pressure is then required to maintain good contact between particles to achieve fast ionic conductivity. Therefore, it is important to understand the impacts of the void space, including space introduced during fabrication and cracks generated over cycling, and to develop strategies for building dense SSBs.

\section{PERSPECTIVES}

The safety issue in lithium-ion systems can be fundamentally addressed by employing solid-state electrolytes to obtain high safety, high energy density, and long calendar lifetime. Apart from searching for new electrolytes with high ionic conductivity, the primary scientific challenge in the development of SSBs is understanding and building controllable solid-state interfaces. 
A
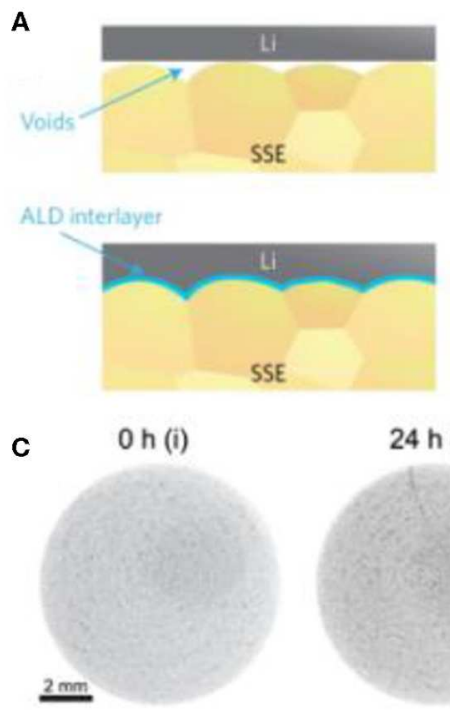

B
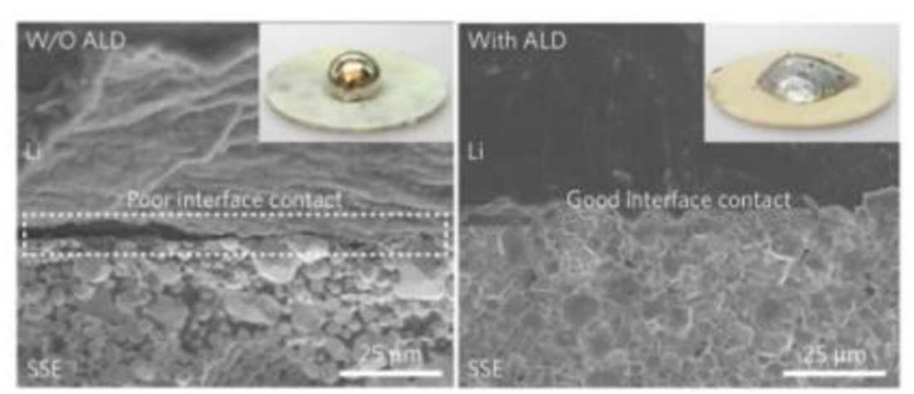

$32 \mathrm{~h}$ (iii)
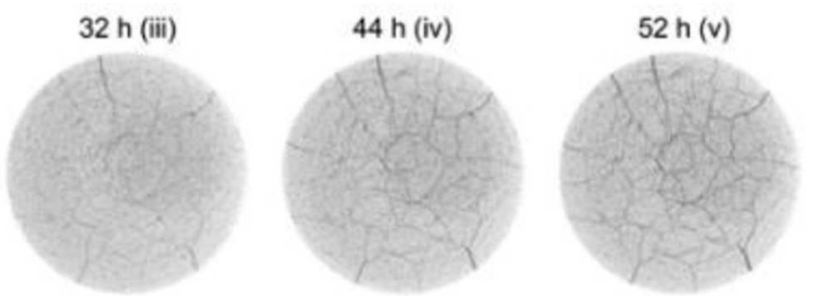

(iii)

(iv)

(v)

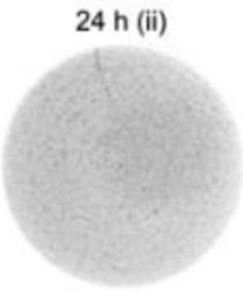

(ii)
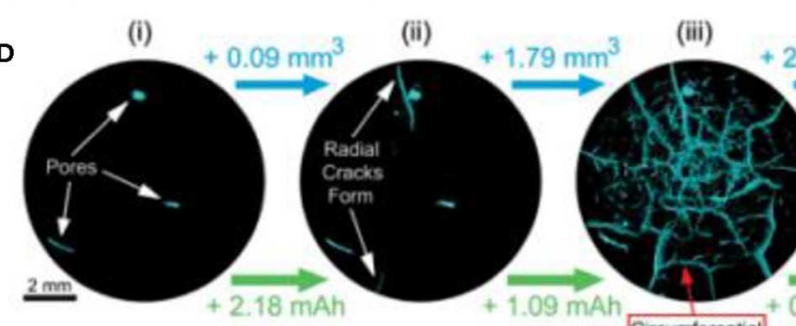

Circumferential
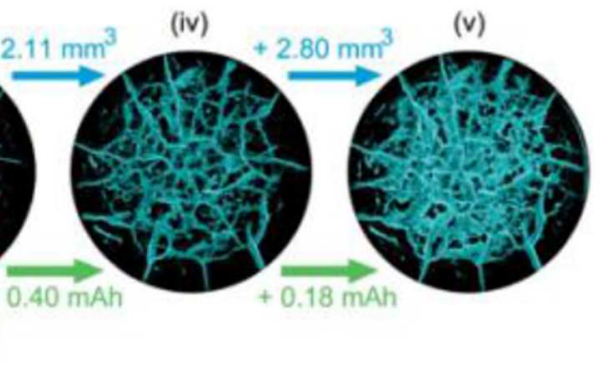

FIGURE 2 | Interface engineering for solid-state electrolytes. (A) Schematic illustrations with and without ALD interlayer coating on SSE and (B) corresponding SEM images. Reproduced from Han et al. (2017) with permission from Nature Publishing Group. (C) Two-dimensional slices extracted from the three-dimensional (3D) tomography of NASICON material $\left(\mathrm{Li}_{1+x} \mathrm{Al}_{x} \mathrm{Ge}_{2-x}\left(\mathrm{PO}_{4}\right)_{3}\right)$ pellet and (D) the 3D crack networks in the pellet. Reproduced from Tippens et al. (2019) with permission from the American Chemical Society.

Many reviews have summarized and highlighted the effects of and exploited strategies for interfaces in SSBs, including electrolyte-electrode interfaces and the interfaces between particles (Wang A. et al., 2018; Famprikis et al., 2019; Lewis et al., 2019). This review points out three main challenges remaining for SSB techniques, regarding the intrinsic features of solid-state electrolytes, the critical interfaces, and the chemomechanical evolution during battery manufacturing and during battery operations. In the future, to further understand and develop SSBs, advanced in-situ and in-operando techniques will provide important knowledge in both scientific and engineering terms (Tripathi et al., 2018). For instance, by coupling electron (Zaefferer, 2011; Wang et al., 2015; Qu et al., 2019), neutron (Ren and Zuo, 2018), and synchrotron-based Xray techniques (Wang L. et al., 2018a,b) with electrochemical measurements, the chemical and structural evolution can be

\section{REFERENCES}

Che, H., Chen, S., Xie, Y., Wang, H., Amine, K., Liao, X.-Z., et al. (2017). Electrolyte design strategies and research progress for room-temperature sodium-ion batteries. Energy Environ. Sci. 10, 1075-1101. doi: 10.1039/C7EE00524E observed from the atomic and nanoscale level to the bulk and even full battery level. Thus, designing in-situ experiments to investigate the accurate chemical and mechanical characteristics of these systems will pave the way to understanding and optimizing SSBs.

\section{AUTHOR CONTRIBUTIONS}

All authors listed have made a substantial, direct and intellectual contribution to the work, and approved it for publication.

\section{FUNDING}

This work was supported by the National Natural Science Foundation of China (51872209 and 51972239) and the Zhejiang Provincial Natural Science Foundation of China (LZ17E020002).

Choi, S., Kim, C., Suh, J. M., and Jang, H. W. (2019). Reduced graphene oxidebased materials for electrochemical energy conversion reactions. Carbon Energy 1, 85-108. doi: 10.1002/cey2.13

de Klerk, N. J. J., and Wagemaker, M. (2016). Diffusion mechanism of the sodium-ion solid electrolyte $\mathrm{Na}_{3} \mathrm{PS}_{4}$ and potential improvements of 
halogen doping. Chem. Mater. 28, 3122-3130. doi: 10.1021/acs.chemmater.6 b00698

Deng, Y., Eames, C., Chotard, J. N., Lalere, F., Seznec, V., Emge, S., et al. (2015). Structural and mechanistic insights into fast lithium-ion conduction in $\mathrm{Li}_{4} \mathrm{SiO}_{4}-\mathrm{Li}_{3} \mathrm{PO}_{4}$ solid electrolytes. J Am. Chem. Soc. 137, 9136-9145. doi: 10.1021/jacs.5b04444

Deng, Y., Eames, C., Fleutot, B., David, R., Chotard, J. N., Suard, E., et al. (2017). Enhancing the lithium ion conductivity in lithium superionic conductor (LISICON) solid electrolytes through a mixed polyanion effect. ACS Appl. Mater. Interfaces 9, 7050-7058. doi: 10.1021/acsami.6b14402

Famprikis, T., Canepa, P., Dawson, J. A., Islam, M. S., and Masquelier, C. (2019). Fundamentals of inorganic solid-state electrolytes for batteries. Nat. Mater. 18, 1278-1291. doi: 10.1038/s41563-019-0431-3

Fan, L., Wei, S., Li, S., Li, Q., and Lu, Y. (2018). Recent progress of the solidstate electrolytes for high-energy metal-based batteries. Adv. Energy Mater. 8:1702657. doi: 10.1002/aenm.201702657

Froboese, L., Sichel, J. F. V. D., Loellhoeffel, T., Helmers, L., and Kwade, A. (2019). Effect of microstructure on the ionic conductivity of an all solid-state battery electrode. J. Electrochem. Soc. 166, A318-A328. doi: 10.1149/2.0601902jes

Han, F., Yue, J., Chen, C., Zhao, N., Fan, X., Ma, Z., et al. (2018). Interphase engineering enabled all-ceramic lithium battery. Joule 2, 497-508. doi: 10.1016/j.joule.2018.02.007

Han, X., Gong, Y., Fu, K. K., He, X., Hitz, G. T., Dai, J., et al. (2017). Negating interfacial impedance in garnet-based solid-state Li metal batteries. Nat. Mater. 16, 572-579. doi: 10.1038/nmat4821

Kato, Y., Shiotani, S., Morita, K., Suzuki, K., Hirayama, M., and Kanno, R. (2018). All-solid-state batteries with thick electrode configurations. J. Phys. Chem. Lett. 9, 607-613. doi: 10.1021/acs.jpclett.7b02880

Kerman, K., Luntz, A., Viswanathan, V., Chiang, Y.-M., and Chen, Z. (2017). Review-practical challenges hindering the development of solid state li ion batteries. J. Electrochem. Soc. 164, A1731-A1744. doi: 10.1149/2.1571707jes

Lewis, J. A., Tippens, J., Cortes, F. J. Q., and Mcdowell, M. T. (2019). Chemomechanical challenges in solid-state batteries. Trends Chem. 1, 845-857. doi: 10.1016/j.trechm.2019.06.013

Li, M., Lu, J., Chen, Z., and Amine, K. (2018). 30 years of lithium-ion batteries. Adv. Mater. 30:e1800561. doi: 10.1002/adma.201800561

Miura, A., Rosero-Navarro, N. C., Sakuda, A., Tadanaga, K., Phuc, N. H. H., Matsuda, A., et al. (2019). Liquid-phase syntheses of sulfide electrolytes for all-solid-state lithium battery. Nat. Rev. Chem. 3, 189-198. doi: 10.1038/s41570-019-0078-2

Nanda, J., Wang, C., and Liu, P. (2018). Frontiers of solid-state batteries. MRS Bull. 43, 740-745. doi: 10.1557/mrs.2018.234

Pfenninger, R., Struzik, M., Garbayo, I., Stilp, E., and Rupp, J. L. M. (2019). A low ride on processing temperature for fast lithium conduction in garnet solid-state battery films. Nat. Energy 4, 475-483. doi: 10.1038/s41560-019-0384-4

Qu, Y., Wang, L., Li, Z., Li, P., Zhang, Q., Lin, Y., et al. (2019). Ambient synthesis of single-atom catalysts from bulk metal via trapping of atoms by surface dangling bonds. Adv. Mater. 31:e1904496. doi: 10.1002/adma.201904496

Ren, Y., and Zuo, X. (2018). Synchrotron X-ray and neutron diffraction, total scattering, and small-angle scattering techniques for rechargeable battery research. Small Methods 2:1800064. doi: 10.1002/smtd.201800064

Strauss, F., Bartsch, T., De Biasi, L., Kim, A. Y., Janek, J., Hartmann, P., et al. (2018). Impact of cathode material particle size on the capacity of bulk-type all-solidstate batteries. ACS Energy Lett. 3, 992-996. doi: 10.1021/acsenergylett.8b00275

Tippens, J., Miers, J. C., Afshar, A., Lewis, J. A., Cortes, F. J. Q., Qiao, H., et al. (2019). Visualizing chemomechanical degradation of a solid-state battery electrolyte. ACS Energy Lett. 4, 1475-1483. doi: 10.1021/acsenergylett.9b00816
Tripathi, A. M., Su, W. N., and Hwang, B. J. (2018). In situ analytical techniques for battery interface analysis. Chem. Soc. Rev. 47, 736-851. doi: 10.1039/C7CS00180K

Wang, A., Kadam, S., Li, H., Shi, S., and Qi, Y. (2018). Review on modeling of the anode solid electrolyte interphase (SEI) for lithium-ion batteries. npj Comput. Mater. 4:15. doi: 10.1038/s41524-018-0064-0

Wang, J., Wang, L., Eng, C., and Wang, J. (2017). Elucidating the irreversible mechanism and voltage hysteresis in conversion reaction for highenergy sodium-metal sulfide batteries. Adv Energy Mater. 7:1602706. doi: 10.1002/aenm.201602706

Wang, L., Wang, J., Guo, F., Ma, L., Ren, Y., Wu, T., et al. (2018b). Understanding the initial irreversibility of metal sulfides for sodium-ion batteries via operando techniques. Nano Energy 43, 184-191. doi: 10.1016/j.nanoen.2017.11.029

Wang, L., Wang, J., Zhang, X., Ren, Y., Zuo, P., Yin, G., et al. (2017). Unravelling the origin of irreversible capacity loss in $\mathrm{NaNiO}_{2}$ for high voltage sodium ion batteries. Nano Energy 34, 215-223. doi: 10.1016/j.nanoen.2017.02.046

Wang, L., Wang, J., and Zuo, P. (2018a). Probing battery electrochemistry with in operando synchrotron X-ray imaging techniques. Small Methods 2:1700293. doi: 10.1002/smtd.201700293

Wang, L., Zuo, P., Yin, G., Ma, Y., Cheng, X., Du, C., et al. (2015). Improved electrochemical performance and capacity fading mechanism of nano-sized LiMn0.9Fe0.1PO4 cathode modified by polyacene coating. J. Mater. Chem. A 3, 1569-1579. doi: 10.1039/C4TA05900J

Wang, S., Xu, H., Li, W., Dolocan, A., and Manthiram, A. (2018). Interfacial chemistry in solid-state batteries: formation of interphase and its consequences. J. Am. Chem. Soc. 140, 250-257. doi: 10.1021/jacs.7b09531

Yuan, Y., and Lu, J. (2019). Demanding energy from carbon. Carbon Energy 1, 8-12. doi: $10.1002 /$ cey 2.12

Zaefferer, S. (2011). A critical review of orientation microscopy in SEM and TEM. Cryst. Res. Technol. 46, 607-628. doi: 10.1002/crat.201100125

Zhang, H., Li, C., Piszcz, M., Coya, E., Rojo, T., Rodriguez-Martinez, L. M., et al. (2017). Single lithium-ion conducting solid polymer electrolytes: advances and perspectives. Chem. Soc. Rev. 46, 797-815. doi: 10.1039/C6CS00491A

Zhang, W., Schröder, D., Arlt, T., Manke, I., Koerver, R., Pinedo, R., et al. (2017). (Electro)chemical expansion during cycling: monitoring the pressure changes in operating solid-state lithium batteries. J. Mater. Chem. A 5, 9929-9936. doi: 10.1039/C7TA02730C

Zhang, Z., Shao, Y., Lotsch, B., Hu, Y.-S., Li, H., Janek, J., et al. (2018). New horizons for inorganic solid state ion conductors. Energy Environ. Sci. 11, 1945-1976. doi: 10.1039/C8EE01053F

Zhao, C., Liu, L., Qi, X., Lu, Y., Wu, F., Zhao, J., et al. (2018). Solid-state sodium batteries. Adv. Energy Mater. 8:1703012. doi: 10.1002/aenm.201703012

Zheng, F., Kotobuki, M., Song, S., Lai, M. O., and Lu, L. (2018). Review on solid electrolytes for all-solid-state lithium-ion batteries. J. Power Sour. 389, 198-213. doi: 10.1016/j.jpowsour.2018.04.022

Conflict of Interest: The authors declare that the research was conducted in the absence of any commercial or financial relationships that could be construed as a potential conflict of interest.

Copyright (C) 2020 Wang, Li, Lu, Li, Tao, Shi, Jin, Chen and Wang. This is an open-access article distributed under the terms of the Creative Commons Attribution License (CC BY). The use, distribution or reproduction in other forums is permitted, provided the original author(s) and the copyright owner(s) are credited and that the original publication in this journal is cited, in accordance with accepted academic practice. No use, distribution or reproduction is permitted which does not comply with these terms. 